\title{
EFECTOS DEL CAMBIO CLIMÁTICO Y DEL MEJORAMIENTO GENÉTICO SOBRE EL RENDIMIENTO DE TRIGO EN EL NORTE DE SANTA FE EN LAS PRIMERAS DOS DÉCADAS DEL SIGLO XXI
}

\section{Effects of climate change and genetic improvement on wheat yield in the North of Santa Fe in the first two decades of the 21st Century}

\author{
Abbate, Pablo E. ${ }^{1}$; Brach, Ana M. ${ }^{2}$ \\ ${ }^{1}$ INTA Balcarce. Balcarce, Buenos Aires, Argentina. abbate.pablo@inta.gob.ar; \\ ${ }^{2}$ INTA Reconquista. Reconquista, Santa Fe, Argentina. brach.ana@inta.gob.ar
}

\section{RESUMEN}

A partir del año 2000 la tasa de aumento de rendimiento $(\boldsymbol{R T O})$ de trigo pan en Argentina alcanzó $46.0 \mathrm{~kg} \mathrm{ha}^{-1}$ año $^{-1}$, mientras que el norte de Santa Fe mantuvo la misma tasa desde los años 1970. Para indagar el origen de la diferencia, se analizó si en las dos últimas décadas hubo algún cambio climático relevante, cuál fue la brecha tecnológica departamental, y cuál fue el aporte del mejoramiento genético al cultivo. Los datos de la estación meteorológica de INTA Reconquista mostraron que el aumento del déficit de presión de vapor ponderado $\left(\boldsymbol{D P V} \boldsymbol{V}_{\boldsymbol{P}}\right)$ promediado desde $20 \mathrm{~d}$ antes a $10 \mathrm{~d}$ después del 1-sep. (fecha óptima de espigazón), fue la variable de cambio climático más desfavorable, entre la serie de años recientes (2000-2018) y previos (1971-1999). Se estimó que al aumento del $\boldsymbol{D P} \boldsymbol{V}_{\boldsymbol{P}}$ correspondería una caída de $16 \%$ en el $\boldsymbol{R T O}$ cuando el nivel de estrés hídrico fue $45 \%$; por lo cual, los cambios climáticos implicarían una reducción del $51 \%$ en la tasa de aumento de $\boldsymbol{R T O}$ entre el norte de Santa Fe. Se encontró que la brecha entre el $\boldsymbol{R T O}$ promedio de la RET-INASE de INTA Reconquista y el promedio departamental fue $66 \%$ y que la brecha respecto de los cultivares de mayor $\boldsymbol{R T O}$ fue $125 \%$. El avance genético se analizó comparando el $\boldsymbol{R T O}$ los cultivares evaluados en la RET con el $\boldsymbol{R T O}$ de cultivares testigos. No se encontró evidencia de avance genético continuo en los últimos 10-13 años. Un mayor avance genético podría impulsar la difusión del cultivo para superar el 22\% de la superficie agrícola del norte de Santa Fe que ocupa actualmente.

Palabras Clave: Cociente fototermal, déficit de presión de vapor, frecuencia de helada tardía, rendimiento potencial, ensayos comparativos de rendimiento, brecha de rendimiento.

\begin{abstract}
Since 2000, the yield increase rate (RTO) of bread wheat in Argentina reached $46.0 \mathrm{~kg} \mathrm{ha}^{-1} \mathrm{yr}^{-1}$, whereas in the Northern area of Santa Fe that rate has remained the same since the 1970s. In order to find out the reason of this contrast, many aspects were considered, such as any relevant climate change in the last two decades, the technological gap between $\boldsymbol{R T O}$ harvest trials and local production and the contribution of crop genetic improvement. Data from INTA Reconquista weather station showed that growth in vapor pressure deficit $\left(\boldsymbol{D P} \boldsymbol{V}_{\boldsymbol{P}}\right)$, averaged from $20 \mathrm{~d}$ to $10 \mathrm{~d}$ after Sep-1 (optimmal date of heading), which was the most disadvantageous weather change variable found, between recent years

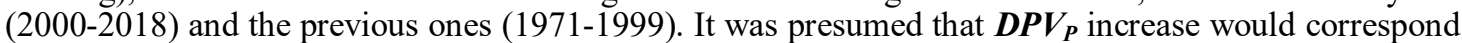
to $16 \%$ drop in $\boldsymbol{R T O}$ when water stress level was $45 \%$; therefore, climatic changes would imply $51 \%$ reduction in $\boldsymbol{R T O}$ rate raise in the North of Santa Fe. It was found that the gap between $\boldsymbol{R T O}$ average in RET-INASE INTA Reconquista and the local average was $66 \%$ and that the gap respect the highest crop $\boldsymbol{R T O}$ was $25 \%$. Genetic advance was analysed comparing the $\boldsymbol{R T O}$ of the cultivars evaluated in the RET with the $\boldsymbol{R T O}$ of cultivars control. No evidence of continuous genetic advance was found in the last 10-13 years. Greater genetic advance could encourage the spread of crop to exceed $22 \%$ of the agricultural area in the North of Santa Fe that is currently occupied.
\end{abstract}

Key words: Photothermal quotient, vapor pressure deficit, late frosts, potential yield, comparative performance trials, yield gap.

Recibido: 20/ene/2020. Aceptado: 24/jun/2020 
Abbate, P.E. y Brach, A.M.: Trigo: cambio climático y mejoramiento genético.

\section{INTRODUCCIÓN}

La superficie sembrada con trigo pan (Triticum aestivum L.) en el norte de la provincia de Santa Fe (correspondiente a los departamentos de 9 de Julio, Gral. Obligado, San Javier y Vera) se incrementó sin discontinuidades relevantes desde el año 2007 hasta la actualidad, aumentando de $27 \cdot 10^{3}$ ha a $145 \cdot 10^{3}$ ha (promedio de las campañas 2016 a 2018; DEAD, 2019). Este último valor representa el $22 \%$ de las $660 \cdot 10^{3}$ ha agrícolas del norte de Santa Fe (calculado a partir de los datos de Giorgi et al., 2009) y el 14\% de la superficie de trigo de la provincia (calculado a partir de los datos de la DEAD, 2019).

La producción de trigo en el norte de Santa Fe se desarrolla en un ambiente de transición entre el clima templado de la región Pampeana y el subtropical de la región Chaqueña. Según los datos registrados en la estación meteorológica de INTA Reconquista durante los últimos 20 años (2000-2019), la precipitación anual varió entre 615 y $1857 \mathrm{~mm}$, de los cuales el $82 \%$ se distribuyen con buena uniformidad desde octubre hasta abril. Si bien entre mediados de otoño e inicios de primavera las precipitaciones son escasas, por lo común alcanzan para recargar a capacidad de campo el perfil del suelo antes del inicio de la siembra de trigo y para mantener un balance de agua positivo hasta mediados de julio. A partir de entonces, aumenta la probabilidad de ocurrencia de estrés hídrico alcanzando su máxima peligrosidad entre la encañazón y la espigazón. Posteriormente, la disponibilidad hídrica suele mejorar, pero aumenta la temperatura media y el riesgo de golpe de calor (temperatura máxima $>32^{\circ} \mathrm{C}$ ) durante el llenado del grano. Esta situación climática, no solo reduce el rendimiento $(\boldsymbol{R T O} \boldsymbol{O})$ del cultivo de manera directa, sino también a través de la menor adopción de tecnologías por parte de los productores, dada la escasa respuesta esperada del cultivo. De hecho, en el "Relevamiento de tecnológico agrícola aplicada" que la Bolsa de Cereales está realizando anualmente, al $85 \%$ de la zona se le asignó un nivel tecnológico medio a bajo (Bolsa de Cereales, 2019). El trigo no es el cultivo dominante de la zona; no obstante, su inclusión en la secuencia agrícola es importante porqué contribuye a la sustentabilidad del sistema agrícola ya que brinda cobertura al suelo y su aporte de rastrojo permite mejorar y preservar las propiedades físicas y químicas del suelo. Además, la producción de trigo le brinda al productor un ingreso financiero al final de la primavera, el cual contribuye a cubrir los gastos de la siembra de los cultivos de verano (principalmente soja). Otro aspecto favorable de la producción local de trigo es que, la mayor parte, se comercializa a través de acopios zonales y es procesada en molinos locales, agregando valor en origen.

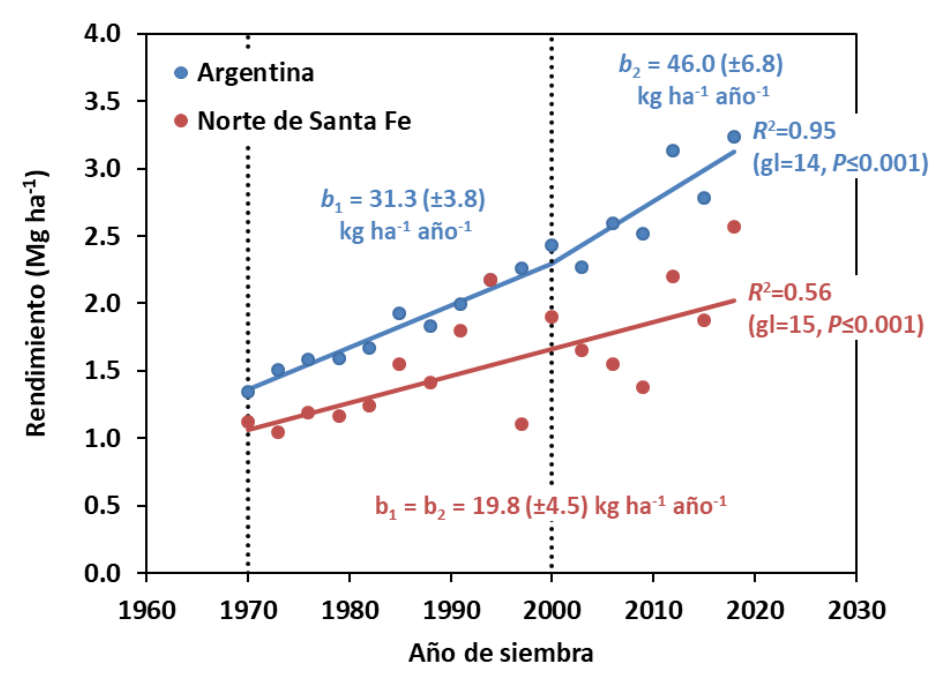

Figura 1. Evolución del rendimiento de trigo (promedio cada tres años) desde el año 1970 a 2018 para Argentina y el norte de la provincia de Santa Fe (dptos. de 9 de Julio, Gral. Obligado, San Javier y Vera). Las líneas de regresión corresponden a un modelo bilineal con primera fase para el período 1970-1999 y segunda para el período 2000-2018 con pendientes $b_{1}, \mathrm{y} b_{2}$ ( \pm error estándar); el modelo bilineal no fue significativo para el norte de Santa Fe. Elaboración propia a partir de datos de la DEAD (2019). 
Hacia 1970 el $\boldsymbol{R T O}$ promedio de Argentina rondaba $1.3 \mathrm{Mg} \mathrm{ha}^{-1}$ y el del norte de la provincia de $\mathrm{Santa} \mathrm{Fe}$ 22\% menor (Fig. 1). Entre los años 1970 y 1999 el $\boldsymbol{R}$ TO promedio de trigo de Argentina se incrementó a razón de $31.3 \mathrm{~kg} \mathrm{ha}^{-1} \mathrm{año}^{-1}$ (Fig. 1). Sin embargo, el incremento en el norte de Santa Fe fue $11.5 \mathrm{~kg} \mathrm{ha}^{-1}$ año $^{-1}$ menor, promediando $19.4 \mathrm{~kg} \mathrm{ha}^{-1}$ año ${ }^{-1}$ (Fig. 1). En consecuencia, al final de este período el $\boldsymbol{R T O}$ de esta zona estaba $28 \%$ por debajo del promedio de Argentina. A partir del año 2000 el aumento promedio del RTO de trigo de Argentina se incrementó respecto del período anterior, alcanzando una tasa de $46.0 \mathrm{~kg} \mathrm{ha}^{-1}$ año ${ }^{-1}$ (Fig. 1). Por su parte, el $\boldsymbol{R} \boldsymbol{T} \boldsymbol{O}$ del norte de Santa Fe mantuvo la misma tasa de aumento que en el período anterior. En consecuencia, actualmente el $\boldsymbol{R}$ TO de esta zona pasó a estar 35\% por debajo del promedio de Argentina. En definitiva, los datos presentados en la Fig. 1 muestran que la brecha de $\boldsymbol{R} \boldsymbol{T} \boldsymbol{O}$ entre el norte de Santa Fe y el promedio nacional se incrementó particularmente en los últimos 20 años. ¿A qué se debió la baja tasa de aumento de rendimiento del norte santafesino? Esto podría ser consecuencia de (i) un efecto de cambios climáticos, (ii) la falta de nuevos cultivares adaptados a la zona, i.e. un bajo progreso genético, (iii) la aplicación de un bajo nivel tecnológico, i.e. una amplia brecha tecnológica, (iv) una combinación de los problemas anteriores. Los objetivos del trabajo fueron: (i) determinar si hubo cambios climáticos que pudieron reducir el aumento de $\boldsymbol{R T O}$ trigo, (ii) estimar la brecha tecnológica del RTO y (ii) evaluar el impacto del mejoramiento genético del trigo, en el norte de Santa FE, durante las dos últimas décadas.

\section{MATERIALES Y MÉTODOS}

\section{Datos meteorológicos}

Los datos meteorológicos diarios de los años 1971 a 2018 fueron obtenidos de la estación meteorológica convencional de la Estación Experimental Agropecuaria INTA Reconquista (2911' S, 59 52’ O, 50 m s.n.m.), departamento de General Obligado, provincia de Santa Fe, correspondiente al norte de la Subregión triguera I. A partir de esos datos se calculó diariamente el cociente fototermal $\left(\boldsymbol{Q}, \mathrm{MJ} \mathrm{m}^{-2} \mathrm{~d}^{-1}{ }^{\circ} \mathrm{C}^{-1}\right.$; Fischer, 1985 ; Abbate, 2017) como:

$$
\text { Ec.[1] } Q=\frac{R / 2}{T-4.5}
$$

donde $\boldsymbol{R}$ es la radiación solar total $\left(\mathrm{MJ} \mathrm{m}^{-2} \mathrm{~d}^{-1}\right), \boldsymbol{R} / \mathbf{2}$ representa la radiación fotosintéticamente activa, $\boldsymbol{T}$ es la temperatura promedio $\left({ }^{\circ} \mathrm{C}\right)$ y 4.5 es la temperatura base de desarrollo $\left({ }^{\circ} \mathrm{C}\right)$, promediando ambas variables desde 20 d precedente y 10 d posteriores a la espigazón. El $\boldsymbol{Q}$ muestra el efecto combinado de la radiación y la temperatura sobre el número de granos por unidad de superficie y se asoció con este, tanto en localidades de Argentina como del resto del mundo (Lázaro y Abbate, 2012).

La evapotranspiración potencial (ETP) se calculó por el método FAO 56 (Allen et al., 1998) a partir de los datos diarios. El déficit de presión de vapor diario ponderado $\left(\boldsymbol{D P} \boldsymbol{V}_{\boldsymbol{P}}, \mathrm{kPa}\right)$ se calculó siguiendo el método de Abbate et al. (2004), a partir de las temperaturas máximas $\left(\boldsymbol{T M A} \boldsymbol{X},{ }^{\circ} \mathrm{C}\right)$ y mínimas $\left(\boldsymbol{T M I N},{ }^{\circ} \mathrm{C}\right)$ diarias, y de la correspondiente humedad relativa media del aire $(\boldsymbol{H R}, \%)$, ponderando la presión de vapor saturada por $\boldsymbol{\Theta}=$ 0.72 , de la siguiente manera:

$$
\begin{aligned}
& \text { Ec.[2] } D P V_{p}=e_{s}-e_{a} \\
& \text { Ec.[3] } e_{a}=R H / 100 \quad e(T M E D) \\
& \text { Ec.[4] } e_{s}=\Theta \quad e(T M A X)+(1-\Theta) e(T M I N) \\
& \text { Ec.[5] } e(T)=0.611 \quad \exp \left[\begin{array}{ll}
(17.27 & T) /(T+237.3)
\end{array}\right]
\end{aligned}
$$

donde $\boldsymbol{e}_{\boldsymbol{s}}$ es la presión de vapor saturado, $\boldsymbol{e}_{\boldsymbol{a}}$ la presión de vapor real y $\boldsymbol{e}(\boldsymbol{T})$ es una función para calcular la presión de vapor a partir de $\boldsymbol{T}=\boldsymbol{T M} \boldsymbol{A} \boldsymbol{X}$ o $\boldsymbol{T}=\boldsymbol{T M I N}$. En ausencia de estrés hídrico, el $\boldsymbol{D P} \boldsymbol{V}_{\boldsymbol{P}}$ se asoció con la eficiencia de uso de la radiación (Kemanian et al., 2004; Cantarero et al., 2016). Por otra parte, el $\boldsymbol{D} \boldsymbol{P} \boldsymbol{V}_{\boldsymbol{P}}$ se asoció a la eficiencia de uso de agua (Tanner y Sinclair, 1983) y de se han validado relaciones entre estas variables en Argentina (Abbate et al., 2004) y Francia (Ghanem et al., 2020) para distintas condiciones hídricas. 
Abbate, P.E. y Brach, A.M.: Trigo: cambio climático y mejoramiento genético.

Los datos medidos en la estación meteorológica y las variables calculadas se promediaron cada 10 días (decenos) para facilitar la comparación entre la serie de los 19 años más recientes (2000-2018) respecto la serie de 29 años previos (1971-1999). Tanto para los años recientes y los previos, se computó la frecuencia de helada tardía (FHT) buscando la fecha de última helada (última fecha del año con $\boldsymbol{T M I N}$ diaria en abrigo meteorológico $\leq 0^{\circ} \mathrm{C}$ ) de cada año. Luego se calculó la $\boldsymbol{F H T}$ como el porcentaje de años que presentaron la última helada con posterioridad a la fecha de última helada de cada año.

A fin integrar el efecto de las variables meteorológicas medidas y calculadas sobre el rendimiento en grano $(\boldsymbol{R T O})$, a partir de los promedios decenarios se estimó el $\boldsymbol{R T O}$ para la serie de años recientes y la serie de años previos, por medio de un modelo, construido a partir de la integración de las siguientes relaciones basadas en variables meteorológicas: (i) una ecuación ajustada y validad por Cantarero et al. (2016) con datos de Balcarce, Córdoba y Paraná, para estimar el número de granos $\mathrm{m}^{-2}(\boldsymbol{N G})$ en función del peso seco de las espigas en antesis y el $\boldsymbol{D P V} \boldsymbol{p}$, (ii) una asociación lineal entre el $\boldsymbol{N} \boldsymbol{G}$ y el $\boldsymbol{Q}$ ajustada por Lázaro y Abbate (2012) a partir de los datos de Argentina, México y Francia y (iii) una asociación lineal entre el peso por grano $(\boldsymbol{P G}, \mathrm{mg})$ y la temperatura promedio durante el llenado del grano, obtenida por Abbate y Lázaro (2010). Las ecuaciones utilizadas fueron:

$$
\begin{aligned}
& \text { Ec.[6] } R T O=P G \frac{N G}{1-0.14} 10^{-5} \\
& \text { Ec.[7] } P G=51-1.2 \quad T_{L L} \\
& \text { Ec.[8] } N G=4 \quad 10^{3}+51 \quad P S E+C C V \quad 10^{3}-\max \left(-10+91 \quad D P V_{p}, 51\right) \quad \triangle P S E \\
& \text { Ec.[9] } P S E=51+13 \frac{10^{3}}{51} Q \\
& \text { Ec.[10] } \triangle P S E=P S E S \quad 10^{-2}
\end{aligned}
$$

donde el $\boldsymbol{R T O}\left(\mathrm{Mg} \mathrm{ha}^{-1}\right)$ se expresó con $14 \%$ de humedad (humedad de comercialización de trigo pan en Argentina, SAGyP, 2004). La $\boldsymbol{T}_{L L}\left({ }^{\circ} \mathrm{C}\right)$ es la temperatura promedio durante los $35 \mathrm{~d}$ posteriores a espigazón. El $\boldsymbol{P S} \boldsymbol{E}\left(\mathrm{g} \mathrm{m}^{-2}\right)$ es el peso seco de las espigas en antesis, sin estrés hídrico; $\triangle \boldsymbol{P S} \boldsymbol{E}\left(\mathrm{g} \mathrm{m}^{-2}\right)$ es la diferencia en el $\boldsymbol{P S} \boldsymbol{E}$ de un cultivo sin estrés hídrico respecto de un cultivo con estrés hídrico. El $\boldsymbol{C C V}$ es un factor del cultivar $(\boldsymbol{C} \boldsymbol{C}=0.9$ para este análisis) y $\boldsymbol{s}$ es el nivel de estrés hídrico expresado como porcentaje de reducción del $\boldsymbol{P S E}$. Para $\boldsymbol{s}=0$, la Ec.[6] a Ec.[10] permite estimar el $\boldsymbol{R} \boldsymbol{T} \boldsymbol{O}$ potencial $\left(\boldsymbol{R T O} \boldsymbol{O}_{P}\right)$, i.e. el $\boldsymbol{R} \boldsymbol{T} \boldsymbol{O}$ esperado sin deficiencias hídricas, ni nutricionales, ni limitaciones sanitarias, a partir del $\boldsymbol{Q}$ y la $\boldsymbol{T}_{\boldsymbol{L} L}$. El $\boldsymbol{R} \boldsymbol{T} \boldsymbol{O}_{\boldsymbol{P}}$ así estimado tuvo una diferencia menor a $2 \%$ respecto de los rendimientos obtenidos con $\boldsymbol{Q}>0.35$, i.e. valores de $\boldsymbol{Q}$ correspondientes a condiciones naturales, por Brach (2011) en experimentos conducidos en condiciones potenciales en INTA Reconquista. Para $\boldsymbol{s}>0$ la Ec.[6] a Ec.[10] permite estimar el $\boldsymbol{R} \boldsymbol{O} \boldsymbol{O}$ considerando el efecto depresor del $\boldsymbol{D P V}_{\boldsymbol{P}}$ y $\boldsymbol{s}$ sobre el $\boldsymbol{R T O} \boldsymbol{O}_{\boldsymbol{P}}$. El valor de $\boldsymbol{s}$ se estimó a partir de la diferencia entre el $\boldsymbol{R T O}$ promedio observado cada año, en cada época de siembra, en los ensayos de la RET-INASE de INTA Reconquista (Tabla 1) y el correspondiente $\boldsymbol{R T O} \boldsymbol{O}_{P}$ estimado; del valor resultante fue $\boldsymbol{s}=45 \%$. Dado que no toda la diferencia entre el $\boldsymbol{R T O}_{\boldsymbol{P}}$ estimado y el $\boldsymbol{R T O}$ obtenido en los ensayos la RET-INASE de INTA Reconquista se debería al efecto del estrés hídrico, es de suponer que el valor asignado a $s$ fue sobreestimado en alguna medida.

\section{Datos de cultivo: manejo de los ensayos y mediciones}

Los datos de cultivo analizados fueron obtenidos en las campañas 2004 a 2018, en los ensayos correspondientes a la Red de ensayos de cultivares de trigo pan coordinada por el Instituto Nacional de Semillas (RETINASE) conducida en INTA Reconquista (Tabla 1). La siembra fue directa, en un suelo Argiudol acuértico (USDA, 2014), de textura franco-limoso en superficie y arcilloso en profundidad (Vidal, 2006). El cultivo antecesor fue girasol. La fertilización consistió en la aplicación $80 \mathrm{~kg} \mathrm{ha}^{-1}$ de fosfato diamónico junto con $60 \mathrm{~kg} \mathrm{ha}^{-1}$ de urea a la siembra y $60 \mathrm{~kg} \mathrm{ha}^{-1}$ de urea en macollaje. El control de malezas se realizó en presiembra con glifosato y en postemergencia temprana con metsulfuron metil, dicamba, fluroxypyr y/o 2,4 D. Cuando la presencia 
de insectos superó el umbral de daño económico, se aplicó una mezcla de insecticidas (Tiametoxam + Lambdacialotrina). Los ensayos se condujeron sin aplicación de fungicida foliar y sin riego. El tamaño de las parcelas sembradas (unidad experimental) fue de $1.4 \mathrm{~m}$ de ancho ( 7 surcos separados a $0.20 \mathrm{~m}$ ) por $5 \mathrm{~m}$ de largo.

En el protocolo de la RET-INASE se establecen las fechas de siembra y la cantidad de plantas a lograr para cada Subregión. Para la Subregión I estas fueron, $1^{\circ}$ época: 10-may, 250 plantas logradas $\mathrm{m}^{-2}$; $2^{\circ}$ época: 30 may, 280; $3^{\circ}$ época: 15 -jun, 300 y $4^{\circ}$ época: 30 jun, 320 . Los cultivares se distribuyeron en las épocas de siembra según su ciclo. La asignación de los cultivares a las épocas de siembra fue a elección del respectivo criadero. Dado que la $1^{\circ}$ época tuvo problemas de implantación en más del $50 \%$ de los años, esta época se descartó del análisis. En la Tabla 1 se presenta la fecha de siembra y la cantidad de cultivares evaluados en cada época, en cada año.

El RTO se midió cosechando mecánicamente los cincos surcos centrales de cada parcela. Los $\boldsymbol{R T O}$ presentados se expresaron con $14 \%$ de humedad transformando el $\boldsymbol{R T O}$ inicial del grano $\left(\boldsymbol{R T O} \boldsymbol{O}_{i}\right)$ por medio de la siguiente ecuación:

$$
\text { Ec.[11] } R T O=R T O_{i} \frac{1-\frac{H_{i}}{100}}{1-0.14}
$$

donde $\boldsymbol{H}_{i}$ es el porcentaje de humedad del grano correspondiente al $\boldsymbol{R} \boldsymbol{T} \boldsymbol{O}_{i}$. Los datos de cultivo aquí analizados, junto con su información complementaria, se encuentran publicados en formato XLSX en la página del INASE: https://www.argentina.gob.ar/inase. Los $\boldsymbol{R} T \boldsymbol{O}$ departamentales y zonales fueron calculados a partir de la producción y superficie cosechada reportada en las estadísticas de la DEAD (2019).

Tabla 1. Cantidad de cultivares evaluados $(\boldsymbol{N})$, fecha de siembra $(\boldsymbol{F S})$ y de espigazón $(\boldsymbol{F E})$, rendimiento promedio (RPRO) y máximo $(\boldsymbol{R M A X})$, mínima diferencia significativa $(\boldsymbol{M D S})$, y nivel de significancia para la interacción cultivar $\mathrm{x}$ año de cada experimento con el experimento del año siguiente, considerando todos los cultivares $(\boldsymbol{I})$ o considerando los cultivares testigo vs. los restantes $\left(\boldsymbol{I}_{T}\right)$; para tres épocas de siembra de la RETINASE de INTA Reconquista, durante las campañas 2004 a 2018.

\begin{tabular}{|c|c|c|c|c|c|c|c|c|c|c|c|c|c|c|c|c|c|c|c|c|c|c|c|c|}
\hline \multirow{4}{*}{$\begin{array}{l}\text { Año } \\
2004\end{array}$} & \multirow{4}{*}{$\begin{array}{l}\mathrm{N} \\
0\end{array}$} & & \multicolumn{6}{|c|}{$2^{\circ}$ época } & \multicolumn{9}{|c|}{$3^{\circ}$ época } & \multicolumn{7}{|c|}{$4^{\circ}$ época } \\
\hline & & & \multicolumn{6}{|c|}{ RPRO RMAX MDS $^{1}$} & \multicolumn{9}{|c|}{ RPRO RMAX MDS $^{1}$} & \multicolumn{7}{|c|}{ RPRO RMAX MDS $^{1}$} \\
\hline & & & $\mathrm{FE}$ & \multicolumn{3}{|c|}{$\left(\mathrm{Mg} \mathrm{ha}^{-1}\right)$} & \multirow{2}{*}{\multicolumn{2}{|c|}{$\begin{array}{ll}\mathrm{I} & \mathrm{I}_{\mathrm{T}} \\
\mathrm{sd} & \mathrm{sd}\end{array}$}} & \multirow{2}{*}{$\begin{array}{l}\mathrm{N} \\
21\end{array}$} & \multirow{2}{*}{$\begin{array}{l}\text { FS } \\
05 / \text { jun. }\end{array}$} & \multirow{2}{*}{$\begin{array}{l}\text { FE } \\
03 / \text { sep. }\end{array}$} & \multicolumn{3}{|c|}{$\left(\mathrm{Mg} \mathrm{ha}^{-1}\right)$} & \multirow{2}{*}{$\begin{array}{l}\text { I } \\
\text { ns }\end{array}$} & \multirow{2}{*}{$\begin{array}{l}\mathrm{I}_{\mathrm{T}} \\
\mathrm{sd}\end{array}$} & \multirow{2}{*}{$\begin{array}{l}\mathrm{N} \\
14\end{array}$} & \multirow{2}{*}{$\begin{array}{l}\text { FS } \\
\text { 07/jul. }\end{array}$} & \multirow{2}{*}{$\begin{array}{l}\text { FE } \\
\text { 23/sep. }\end{array}$} & \multicolumn{3}{|c|}{$\left(\mathrm{Mg} \mathrm{ha}^{-1}\right)$} & \multirow{2}{*}{\multicolumn{2}{|c|}{$\begin{array}{ll}\mathrm{I} & \mathrm{I}_{\mathrm{T}} \\
* & \mathrm{sc}\end{array}$}} \\
\hline & & sd & sd & sd & sd & $\mathrm{sd}$ & & & & & & 2.06 & 2.75 & 0.35 & & & & & & 2.49 & 3.09 & 0.31 & & \\
\hline 2005 & 24 & 26/may. & 09/sep. & 3.24 & 4.52 & 1.39 & $*$ & sd & 27 & $05 /$ jun. & 30/ago. & 3.58 & 4.33 & 0.58 & $*$ & sd & 20 & 04/jul. & $24 /$ sep. & 3.15 & 3.92 & 0.37 & $7 \mathrm{sd}$ & $\mathrm{sd}$ \\
\hline 2006 & 29 & 23/may. & 11/sep. & 1.80 & 2.32 & 0.62 & ns & ns & 29 & 13/jun & 07/sep. & 1.92 & 2.57 & 0.53 & $*$ & sd & 0 & sd & sd & sd & sd & $\mathrm{Sd}$ & sd & sd \\
\hline 2007 & 24 & 17/may. & 12/sep. & 3.01 & 3.93 & 0.46 & sd & ns & 20 & 04/jun & $12 /$ sep. & 2.64 & 3.45 & 0.24 & $*$ & sd & 14 & $27 /$ jun & $21 /$ sep. & 3.31 & 3.87 & 0.28 & $3 *$ & sd \\
\hline 2008 & 25 & 16/may. & 06/sep. & 2.06 & 2.61 & sn & sd & sd & 26 & 30/may & 18/ago. & 2.26 & 3.13 & 0.51 & $*$ & sd & 23 & 26/jun & $10 /$ sep. & 2.54 & 3.21 & 0.49 & $*$ & sd \\
\hline 2009 & 0 & $\mathrm{sd}$ & $\mathrm{sd}$ & sd & $\mathrm{sd}$ & sd & $\mathrm{sd}$ & sd & 28 & $01 /$ jun & 29/ago. & 2.70 & 3.89 & 0.42 & $* 1$ & ns & 23 & $01 /$ jul & 12/sep. & 2.92 & 3.57 & 0.42 & $2 *$ & $\mathrm{sd}$ \\
\hline 2010 & 22 & 08/jun. & $21 /$ sep. & 5.44 & 6.38 & 0.26 & $*$ & ns & 30 & 08/jun & 09/sep. & 4.91 & 5.48 & 0.52 & $* 1$ & ns & 26 & 28/jun & $21 /$ sep. & 5.47 & 6.48 & 0.55 & $5 *$ & ns \\
\hline 2011 & 21 & $02 /$ jun. & 16/sep. & 3.25 & 4.20 & 0.27 & $*$ & $*$ & 25 & $05 /$ jun & $02 /$ sep. & 3.85 & 4.24 & 0.34 & $* 1$ & ns & 21 & $27 /$ jun & $14 /$ sep. & 3.32 & 3.97 & 0.27 & $7 *$ & ns \\
\hline 2012 & 23 & 04/jun. & $14 /$ sep. & 3.09 & 3.93 & 0.29 & $*$ & ns & 22 & 04/jun & 28/ago. & 2.99 & 3.60 & 0.29 & $* 1$ & ns & 18 & 26/jun & 08/sep. & 3.73 & 4.43 & 0.28 & $3 *$ & $\mathrm{~ns}$ \\
\hline 2013 & 24 & $02 /$ jun. & 11/sep. & 2.81 & 3.36 & 0.35 & $*$ & ns & 22 & $02 /$ jun & $02 /$ sep. & 2.13 & 3.08 & 0.28 & $* 1$ & ns & 17 & $25 /$ jun & 11/sep. & 2.83 & 3.45 & 0.30 & $*$ & $\mathrm{~ns}$ \\
\hline 2014 & 25 & 29/may. & 07/sep. & 3.14 & 4.79 & 0.60 & $*$ & ns & 21 & 08/jun & 26/ago. & 2.51 & 3.26 & 0.57 & $*_{1}$ & ns & 15 & 29/jun & $12 /$ sep. & 2.07 & 3.00 & 0.29 & $*$ & ns \\
\hline 2015 & 28 & 31/may. & 07/sep. & 2.15 & 3.48 & 0.54 & $*$ & ns & 30 & 04/jun & 20/ago. & 2.80 & 3.86 & 0.47 & $* 1$ & ns & 20 & 05/jul & $14 /$ sep. & 2.76 & 4.05 & 0.49 & $*$ & $\mathrm{~ns}$ \\
\hline 2016 & 24 & 31/may. & 13/sep. & 4.11 & 4.76 & 0.54 & $*$ & $*$ & 25 & 06/jun & $05 /$ sep. & 4.25 & 4.65 & 0.51 & $*_{1}$ & ns & 18 & $30 /$ jun & 20/sep. & 4.16 & 4.84 & 0.48 & $3 *$ & $\mathrm{~ns}$ \\
\hline 2017 & 30 & 05/jun. & $15 /$ sep. & 2.01 & 2.95 & 0.25 & $*$ & $*$ & 27 & 05/jun & 30/ago. & 2.44 & 3.44 & 0.30 & $* 1$ & ns & 19 & 03/jul & 13/sep. & 2.36 & 3.21 & 0.22 & $2 *$ & $\mathrm{~ns}$ \\
\hline 2018 & 27 & 20/may. & 08/sep. & 4.04 & 4.70 & 0.41 & & & 30 & $05 /$ jun & 06/sep. & 4.08 & 4.62 & 0.47 & & & 21 & $01 / j u l$ & 20/sep. & 2.90 & 3.56 & 0.34 & & \\
\hline Pro. & 25 & $28 /$ may & 12/sep. & 3.09 & 3.99 & 0.50 & & & 26 & 04/jun & 1/sep. & 3.01 & 3.76 & 0.42 & & & 19 & 29/jun & 16/sep. & 3.14 & 3.90 & 0.36 & & \\
\hline
\end{tabular}

${ }^{1}$ MDS: mínima diferencia significativa para comparar cultivares dentro de cada experimento sd: sin dato; ns: no significativo $(P>0.05) ;{ }^{*}$ significativo $(P \leq 0.05)$.

\section{Análisis de los datos}

Cada época de siembra, de cada año, constituyó un experimento estadísticamente independiente, con los cultivares (tratamientos) dispuestos en un diseño en bloques completos aleatorizados, con tres repeticiones. Los datos de cada experimento fueron analizados a través de un análisis de varianza $(A N V A)$ que incluyó el efecto de los tratamientos como factor fijo (Steel y Torrie, 1980). Dado que no todos los cultivares estuvieron presen- 
Abbate, P.E. y Brach, A.M.: Trigo: cambio climático y mejoramiento genético.

tes en los experimentos de todos los años, para evaluar la interacción cultivar $\mathrm{x}$ año (ambiente) en cada época de siembra, se construyó un $A N V A$ combinando experimentos (McIntosh, 1983), que incluyó el efecto de todos los cultivares que participaron en dos años consecutivos (bienio) y la interacción cultivar $\mathrm{x}$ años. Para establecer qué cultivares presentaron diferencias de RTO, se utilizó el método de la mínima diferencia significativa (MDS) de Fisher cuando el $A N V A$ indicó diferencias entre cultivares $(P \leq 0.05)$ (Steel y Torrie, 1980).

Para cada año, la brecha tecnológica promedio (BPRO) se calculó como:

$$
\mathrm{Ec}[12] B P R O_{i}=R P R O_{i}-R D P T O_{i}
$$

donde $\boldsymbol{R} \boldsymbol{P R \boldsymbol { O }} \boldsymbol{O}_{i}$ es el $\boldsymbol{R} \boldsymbol{T} \boldsymbol{O}$ promedio de todos los cultivares evaluados en año $\boldsymbol{i}$ y $\boldsymbol{R D P T \boldsymbol { O } _ { i }}$ es el correspondiente $\boldsymbol{R} \boldsymbol{T O}$ departamental. De manera equivalente se calculó la brecha tecnológica máxima $\boldsymbol{B M} \boldsymbol{A} \boldsymbol{X}_{\boldsymbol{i}}$ del año $\boldsymbol{i}$, como la diferencia entre el $\boldsymbol{R T O}_{i}$ del cultivar que presentó el $\boldsymbol{R} T \boldsymbol{O}_{i}$ máximo $\left(\boldsymbol{R M A X _ { i }}\right)$ y el $\boldsymbol{R D P T O \boldsymbol { O } _ { i }}$.

Para estimar el aumento del RTO atribuible al aporte del mejoramiento genético a través de los años (avance genético), el RTO promedio de cada experimento, se expresó como la diferencia respecto del RTO promedio de 2-3 cultivares testigos que estuvieron presentes en la misma época de siembra en todos los años:

$$
\text { Ec.[13] } D R P R O_{i}=R P R O_{i}-R T_{i}
$$

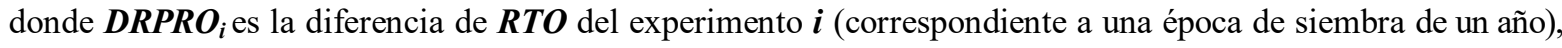
$\boldsymbol{R} \boldsymbol{P R \boldsymbol { O } _ { i }}$ es el $\boldsymbol{R} \boldsymbol{T} \boldsymbol{O}$ promedio de todos los cultivares evaluados en el experimento $\boldsymbol{i}$ y $\boldsymbol{R} \boldsymbol{T}_{i}$ es el $\boldsymbol{R} \boldsymbol{T} \boldsymbol{O}$ promedio de los cultivares testigos en el experimento $\boldsymbol{i}$. El $\boldsymbol{R} \boldsymbol{T}_{i}$ es un estimador del ambiente de cada época en cada año, por lo tanto, la magnitud de $\boldsymbol{D P R \boldsymbol { O } _ { i }}$ puede atribuirse a la superioridad genética de los cultivares participantes del promedio respecto de los cultivares testigos. De manera equivalente se calculó la diferencia $\boldsymbol{D R M A} \boldsymbol{X}_{\boldsymbol{i}}$ entre el $\boldsymbol{R} \boldsymbol{T} \boldsymbol{O}_{i}$ del cultivar que presentó el $\boldsymbol{R} \boldsymbol{M} \boldsymbol{A} \boldsymbol{X}_{\boldsymbol{i}}$ en el ensayo $\boldsymbol{i}$ y el $\boldsymbol{R} \boldsymbol{T}_{\boldsymbol{i}}$.

Para cada cultivar (denotado por el subíndice $i$ ) se calculó la $\boldsymbol{D P R O}_{\boldsymbol{i}}$ (la diferencia de $\boldsymbol{R T O}$ promedio), a través de los $n$ experimentos en que fue evaluado, como:

$$
\operatorname{Ec}[14] D R T O_{i}=\left(\sum_{\mathrm{j}=\mathrm{T}^{\mathrm{j}}=\mathrm{n}}^{\mathrm{n}} R T O_{i j}-R T_{\mathrm{j}}\right) \mathrm{j}
$$

A tal efecto, se consideraron solamente los cultivares evaluados en tres o más años en una misma época de siembra. La $\boldsymbol{D} \boldsymbol{R T O} \boldsymbol{O}_{i}$ así calculada se utilizó para identificar cuáles fueron los cultivares de $\boldsymbol{R} \boldsymbol{T} \boldsymbol{O}$ más destacados pese a haber sido evaluados en distintos años.

Los cultivares testigos elegidos estuvieron presentes la mayor cantidad de años posibles a partir del último (Tabla 2). Los testigos de la $2^{\circ}$ época estuvieron presentes desde el año 2006 a 2018; la inclusión de un tercer cultivar testigos hubiera implicado tres años menos en el análisis. Los testigos para la $3^{\circ}$ y $4^{\circ}$ época estuvieron presentes a partir del año 2009. El avance genético se calculó de dos maneras, como pendiente de la asociación entre (i) la $\boldsymbol{D P R O}_{\boldsymbol{i}}$ o $\boldsymbol{D} \boldsymbol{M} \boldsymbol{A} \boldsymbol{X}_{\boldsymbol{i}}$ y el año de evaluación (método tradicional), y (2) la $\boldsymbol{D} \boldsymbol{R} \boldsymbol{T} \boldsymbol{O}$ y el año de inscripción de cada cultivar (método alternativo propuesto por Fischer et al., 2014).

Tabla 2. Principales características de los cultivares testigos, año de inscripción, primer año de participación en

\begin{tabular}{|c|c|c|c|c|c|}
\hline Cultivar & Inscripción & Primer año & $2^{\circ}$ época & $3^{\circ}$ época & $4^{\circ}$ época \\
\hline KLEIN PROTEO & 2003 & 2004 & $\mathrm{X}$ & & \\
\hline ACA 315 & 2006 & 2006 & $\mathrm{X}$ & & \\
\hline KLEIN TAURO & 2005 & 2005 & & $\mathrm{X}$ & $\mathrm{X}$ \\
\hline BIOINTA 1006 & 2009 & 2009 & & $\mathrm{X}$ & $\mathrm{X}$ \\
\hline KLEIN NUTRIA & 2009 & 2009 & & $\mathrm{X}$ & $\mathrm{X}$ \\
\hline
\end{tabular}
la RET-INASE de INTA Reconquista (todos los testigos estuvieron presentes hasta el año 2018) y época de siembra para la cual fue considerado testigo. 


\section{RESULTADOS Y DISCUSIÓN}

\section{Efectos de los cambios climáticos}

De acuerdo con los datos de la estación meteorológica de INTA Reconquista, la temperatura máxima diaria media (Fig. 2a) fue la temperatura que presentó las mayores diferencias entre los años recientes (2000-2018) y los anterior (1971-1999). Excepto para los dos primeros decenos de mayo, los valores de los años recientes fueron mayores o iguales que en los años anteriores. Incluso se encontró un aumento de la temperatura máxima diaria, promediada desde $20 \mathrm{~d}$ anteriores a $10 \mathrm{~d}$ posteriores a espigazón o promediada durante el llenado del grano, a través de los años $(1971-2018)$ de $0.04 \pm 0.01^{\circ} \mathrm{C}$ año ${ }^{-1}\left(R^{2}=0.12\right.$ y $\left.0.13, \mathrm{gl}=46, P \leq 0.001\right)$. No obstante, la mayor diferencia entre los años recientes y los anteriores, correspondiente al primer deceno de julio, no superó $10 \%\left(1.8^{\circ} \mathrm{C}\right)$ y la diferencia promedio durante el período de cultivo de trigo (1-may. a 31-dic.) fue solo $2 \%\left(0.5^{\circ} \mathrm{C}\right)$ (Fig. 2a). La radiación (Fig. 2b) de los años recientes fue igual o mayor que la de la serie anterior; sin embargo, la diferencia promedio para el período de cultivo de trigo fue de solo $2 \%\left(0.3 \mathrm{MJ} \mathrm{m}^{-2} \mathrm{~d}^{-1}\right)$. El $\boldsymbol{Q}$ (Fig. 2c) presentó la mayor diferencia entre las series de años en el primer deceno de agosto, alcanzando $14 \%$ $\left(0.07 \mathrm{MJ} \mathrm{m}^{-2} \mathrm{~d}^{-1}{ }^{\circ} \mathrm{C}^{-1}\right)$; no obstante, esos cambios son de escasa relevancia ya que es poco factible que la espigazón ocurra antes del 1-sep. Para las fechas de espigazón posteriores al 1-sep. la diferencia promedio de $\boldsymbol{Q}$ entre las series de años fue $0 \%$.

Las diferencias de precipitación (Fig. 2d) entre las series de años fueron oscilantes. En junio y fines de octubre, la precipitación de los años recientes se redujo; sin embargo, considerando todo el período de cultivo de trigo (1-may. a 31-dic.), hubo un aumento de $5 \%(32 \mathrm{~mm})$ a favor de los años recientes. Tampoco se encontró una tendencia clara de la precipitación mensual a través de los años (1971-2018); el único mes con asociación estadísticamente significativa fue septiembre $\left(R^{2}=0.09, \mathrm{gl}=46, P \leq 0.03\right)$ indicando una reducción de $0.76 \pm 0.36 \mathrm{~mm}_{\text {año }}{ }^{-1}$. Con la excepción de unos pocos decenos, la $\boldsymbol{E T P}$ (Fig. 2d) de los años recientes fue levemente mayor que la de los años previos, promediando un aumento de $3 \%\left[1 \mathrm{~mm}(10 \mathrm{~d})^{-1}\right]$.

El $\boldsymbol{D P} \boldsymbol{V}_{\boldsymbol{P}}$ (Fig. 2e) fue la variable meteorológica que presentó la mayor diferencia entre las dos series de años. Hubo solamente cuadro decenos en que los años recientes tuvieron un aumento menor al $5 \%$. El aumento promedio para el período de cultivo de trigo fue $12 \%(0.1 \mathrm{kPa})$, originado en el aumento de la temperatura combinado con una disminución de la humedad relativa de $4 \%$. El $\boldsymbol{D} \boldsymbol{P} \boldsymbol{V}_{\boldsymbol{P}}$ promediado desde $20 \mathrm{~d}$ antes a $10 \mathrm{~d}$ después del 1-sep. (fecha optima de espigazón), fue la variable meteorológica con mayor tasa de cambio a través de los años (1971-2018), aumentando $0.008 \pm 0.003 \mathrm{kPa}^{2} \tilde{n}^{-1}\left(R^{2}=0.15, \mathrm{gl}=46, P \leq 0.01\right)$. Pudo estimarse que al aumento del $\boldsymbol{D P} \boldsymbol{V}_{\boldsymbol{P}}$ de los años recientes le correspondería una caída de $12 \%\left(0.8 \mathrm{~g} \mathrm{~m}^{-2} \mathrm{~mm}^{-1}\right)$ en la eficiencia de uso de agua transpirada.

La $\boldsymbol{F H T}$ de la serie de años recientes presentó diferencias respecto de la $\boldsymbol{F H T}$ de la serie de años previos (Fig. 2f). No obstante, las fechas para un riesgo de heladas entre 5 y $20 \%$, tuvo una diferencia menor a $4 \mathrm{~d}$ entre las dos series de años (Fig. 2f). Según la curva de los años recientes, para que el riesgo de daño por helada en espigazón sea $\leq 10 \%$, esta debería ser posterior al 1-sep.

La Fig. 3 sintetiza el efecto de las variaciones meteorológicas descriptas sobre el $\boldsymbol{R T O} \boldsymbol{O}_{\boldsymbol{P}}$ y el $\boldsymbol{R T O}$ bajo un estrés hídrico de $45 \%$. En la mayor parte de las localidades trigueras de Argentina, el $\boldsymbol{Q}$ decrece de invierno a verano; sin embrago, la (Fig. 2c) muestra que en Reconquista el $\boldsymbol{Q}$ aumenta hasta 1-sep., fecha de para la cual la $\boldsymbol{F H T}$ es $\leq 10 \%$ (Fig. 2f), y luego permanece estable. El comportamiento del $\boldsymbol{Q}$ en Reconquista es similar al de otras localidades de la ribera del río Paraná como Paraná, Corrientes, Encarnación, y Ciudad del Este (Caviglia et al., 2001; Abbate y Lázaro, 2010). No obstante, si se tiene en cuenta que con el retraso de la fecha de espigazón aumentará la temperatura durante el llenado del grano, es de esperar que el $\boldsymbol{R T}_{\boldsymbol{T}} \boldsymbol{O}_{\boldsymbol{P}}$ decaiga tal como lo muestra la Fig. 3. Por tal motivo, desde el punto de vista del $\boldsymbol{R} \boldsymbol{T} \boldsymbol{O}_{\boldsymbol{P}}$, la fecha de espigazón óptima será la más próxima a la fecha para la cual la $\boldsymbol{F H T}$ es $\leq 10 \%$ (u otro valor de riesgo que se quiera adoptar), tal como ocurre en la mayoría de las localidades trigueras. Esta fecha es válida independientemente del ciclo del cultivar. La Fig. 3 muestra que al retrasar la espigazón del 1-sep. al 1-oct., hubo una caída de $\boldsymbol{R T O}$ (calculada como la pendiente de la recta de regresión) de $18 \pm 1$ y $14 \pm 2 \mathrm{~kg} \mathrm{ha}^{-1}$ por d de atraso $(P \leq 0.01$ y $P \leq 0.04$, respectivamente) al pasar de los años anteriores a los recientes. La Fig. 3 también muestra que, durante septiembre, el $\boldsymbol{R T T}_{\boldsymbol{P}}$ de la serie de años recientes resultó $3 \%\left(0.18 \mathrm{Mg} \mathrm{ha}^{-1}\right)$ menor que el de la serie de años anteriores. 


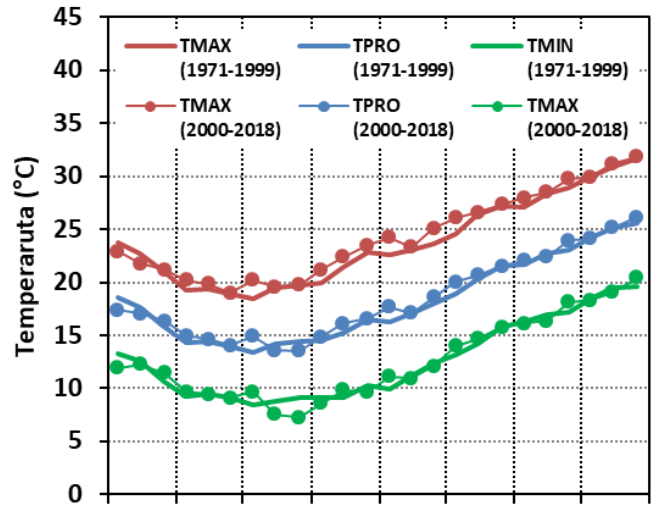

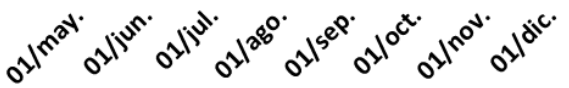
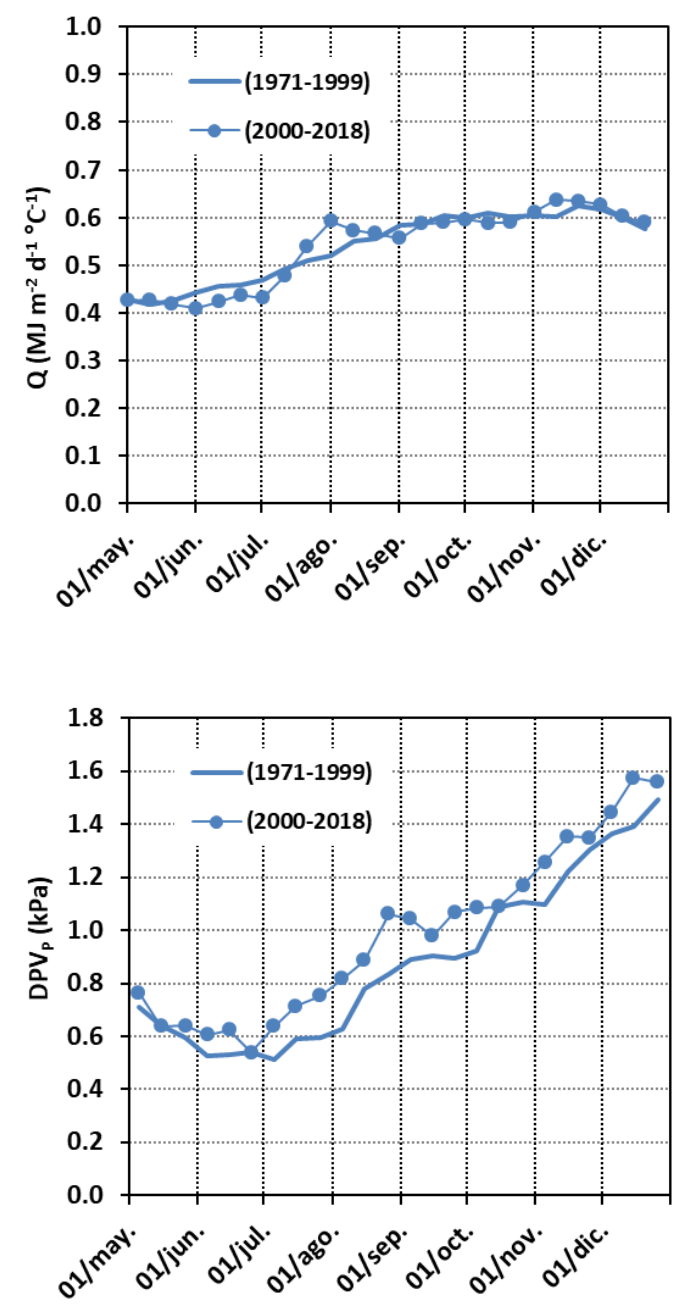

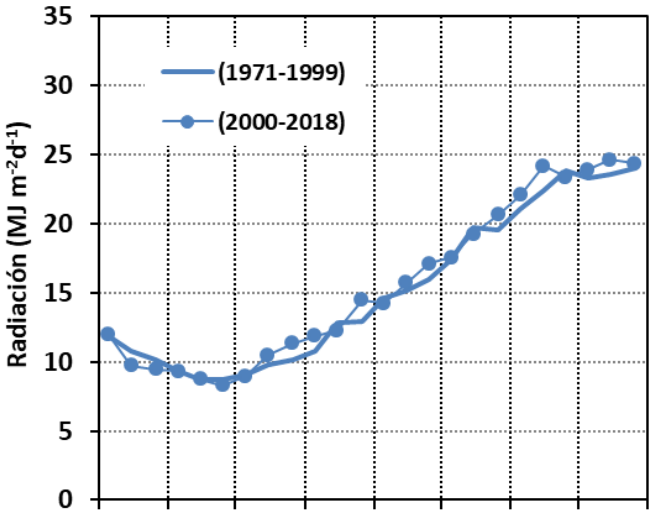

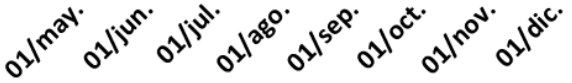
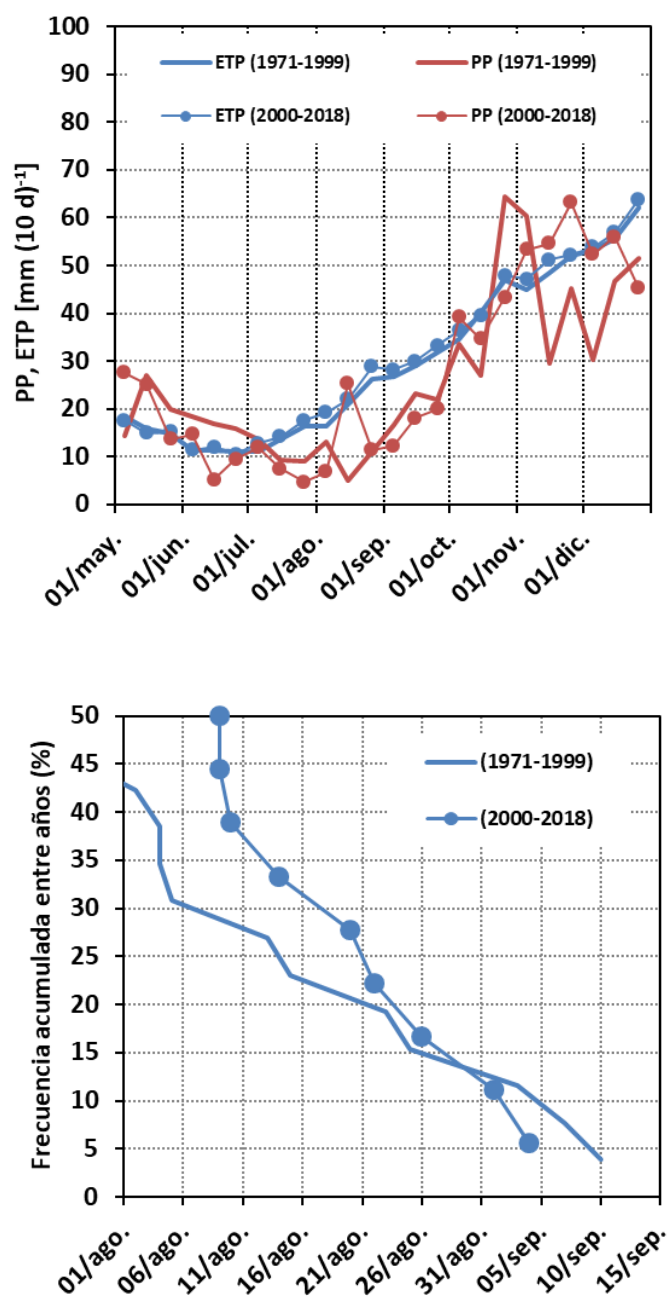

Figura 2. (a) Temperatura máxima (TMAX), mínima (TMIN) y promedio entre TMAX y TMIN (TPRO) (b) radiación solar, (c) cociente fototermal $(\boldsymbol{Q}$, Ec. [1]), (d) precipitación $(\boldsymbol{P P})$ y evapotranspiración potencial $(\boldsymbol{E T P})$ calculada por el método FAO 56 y (e) déficit de presión de vapor ponderado (DPV $\boldsymbol{V}_{\boldsymbol{P}}$, Ec.[2] a Ec.[5]) y (f) frecuencias de helada tardía (TMIN diaria en abrigo meteorológico $<0{ }^{\circ} \mathrm{C}$ ); para la serie 1971-1999 (línea llena) y 2000-2018 (línea con puntos); según datos de la estación meteorológica de INTA Reconquista; datos promediados cada $10 \mathrm{~d}$ excepto la frecuencia de heladas. 
Al considerar el $\boldsymbol{R}$ TO estimado con estrés hídrico (Fig. 3) también se encontró una caída al retrasar la espigazón del 1-sep. al 1-oct., la cual resultó similar para los años recientes y anterior, promediando $14 \pm 3 \mathrm{~kg} \mathrm{ha}^{-1}$ $\mathrm{d}^{-1}(P \leq 0.01)$. Así, desde el punto de vista del $\boldsymbol{R} \boldsymbol{T} \boldsymbol{O}$ bajo estrés hídrico, la fecha de espigazón óptima resulta similar a la establecida a partir del $\boldsymbol{R} T \boldsymbol{O}_{P}$, i.e. la correspondiente a la $\boldsymbol{F H T} \leq 10 \%$, ya que esa fecha presenta la mejor combinación de temperatura (Fig. 2a), radiación (Fig. 2b) y $\boldsymbol{D} \boldsymbol{P} \boldsymbol{V}_{\boldsymbol{P}}$ (Fig. 2e) para maximizar el $\boldsymbol{R T O}$. Por otra parte, según la Fig. 3, para espigazón entre el 1-sep. y el 1-oct., el $\boldsymbol{R} T \boldsymbol{O}$ de los años recientes promedió $16 \%\left(0.50 \mathrm{Mg} \mathrm{ha}^{-1}\right)$ menos que el de los años anteriores. Esta diferencia, que resultó mayor a la encontrada para el $\boldsymbol{R} \boldsymbol{T} \boldsymbol{O}_{\boldsymbol{P}}$, puede atribuirse al aumento del $\boldsymbol{D P} \boldsymbol{V}_{\boldsymbol{P}}$ ya mencionado (Fig. 2e) en los años recientes.

Fischer et al. (2014) calcularon que la tasa de aumento de $\boldsymbol{R} \boldsymbol{T O}$ de trigo a nivel mundial fue $29.8 \mathrm{~kg} \mathrm{ha}^{-1}$ año $^{-1}$ entre los años 1991 a 2010, valor que resulta igual $(P>0.05)$ al de Argentina entre los años 1971-1999 (Fig. 1) y menor $(P \leq 0.05)$ a la de los años 2000-2018 (Fig. 1). Por su parte, en el norte de Santa Fe, la tasa de aumento de $\boldsymbol{R} \boldsymbol{T} \boldsymbol{O}$ entre los años 1971-2018 fue $19.8 \mathrm{~kg} \mathrm{ha}^{-1}$ año ${ }^{-1}$ (Fig. 1). Si al RTO del norte de Santa Fe se le suma el $16 \%$ de pérdida correspondiente a los años recientes en secano, la tasa de aumento llegaría a 41.2 $\mathrm{kg} \mathrm{ha}^{-1}$ año $^{-1}$ valor que iguala estadísticamente $(P>0.05)$ al promedio nacional de los 19 años recientes. No obstante, esta última comparación supondría que el cambio climático afectó solamente al norte de Santa Fe y no al resto de región triguera argentina lo cual es un supuesto poco realista.

En síntesis, los datos meteorológicos de los últimos 19 años presentan algunas características más desfavorables que los años previos, afectando más el $\boldsymbol{R} \boldsymbol{T} \boldsymbol{O}$ bajo estrés hídrico que el $\boldsymbol{R} \boldsymbol{T} \boldsymbol{O}_{\boldsymbol{P}}$. Puede estimarse que la tasa de aumento de $\boldsymbol{R} \boldsymbol{T} \boldsymbol{O}$ real $\left(19.8 \mathrm{~kg} \mathrm{ha}^{-1}\right.$ año $\left.^{-1}\right)$ se redujo hasta $51 \%$ [= $(1-41.2 / 19.4)$ x 100$]$ por efecto de cambios en las variables climáticas.

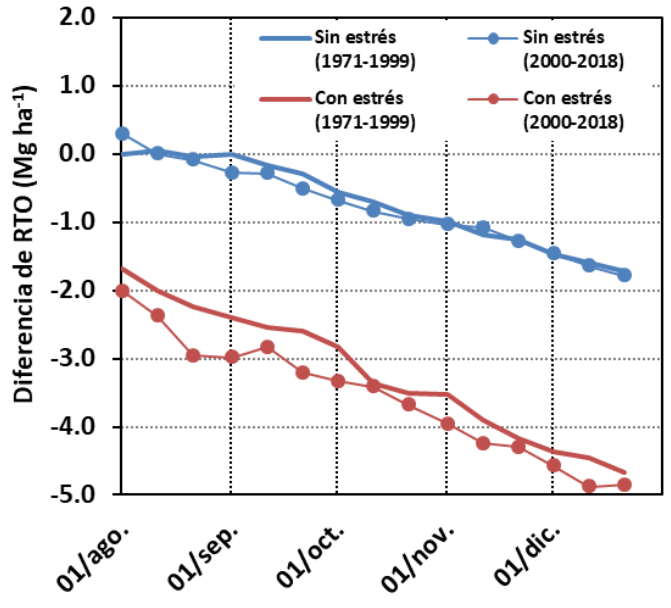

Figura 3. Diferencia de rendimiento (RTO) de trigo estimado para un cultivo sin estrés hídrico y con un estrés hídrico de 45\%, por medio de la Ec.[6] a Ec.[10], a partir de la temperatura, la radiación y el déficit de presión de vapor, en función de la fecha de espigazón; para la serie de años 1971-1999 (línea llena) y 2000-2018 (línea con puntos); según datos de la estación meteorológica de INTA Reconquista. El RTO de referencia es el estimado para la serie 1971-1999, sin estrés y fecha de floración el 1-sep.

\section{Efectos del mejoramiento genético}

En todos los experimentos, excepto en la $2^{\circ}$ época de la campaña 2008 hubo diferencias entre cultivares $(P$ $\leq$ 0.05; Tabla 1). La Fig. 4a y Fig. 4b muestran que, entre los años 2006 a 2018, el $\boldsymbol{R} \boldsymbol{P R \boldsymbol { O }}$ y el $\boldsymbol{R} \boldsymbol{M} \boldsymbol{A} \boldsymbol{X}$ de todas las épocas de siembra de la RET-INASE de INTA Reconquista, fue más fluctuante que el RTO del departamento de Gral. Obligado, con un marcado pico de $\boldsymbol{R} \boldsymbol{T O}$ en el años 2010. Ese año se destacó por presentar una temperatura promedio entre el 1-ago. y el 31-oct. (período que abarca las etapas reproductivas del trigo) de 18.6 ${ }^{\circ} \mathrm{C}$, la cual fue la más baja desde el año 1975. Dada las variaciones meteorológicas entre años, no sorprende que la asociación entre el $\boldsymbol{R} \boldsymbol{T} \boldsymbol{O}$ y el año de siembra hayan resultado bajas $(P>0.05)$ para todas las época de siembra. Por su parte, las regresiones recíprocas entre épocas resultaron altas $\left(R^{2}>0.85 ; P \leq 0.01\right)$ tanto para el $\boldsymbol{R P R O}$ como para el $\boldsymbol{R M A X}$, indicando que el clima no presentó un efecto diferencial entre épocas, si bien existieron diferencias propias del año como es el caso del $\boldsymbol{R} \boldsymbol{M} \boldsymbol{A} \boldsymbol{X}$ en el año 2014 (Fig. 4b). Otro aspecto 
Abbate, P.E. y Brach, A.M.: Trigo: cambio climático y mejoramiento genético.

que surge de la Fig. 4a, la Fig. 4b y de la Tabla 1 es que los cultivares de ciclo intermedio sembrados a fines de mayo ( $2^{\circ}$ época), los ciclos intermedio-cortos sembrados a mediados de junio ( $3^{\circ}$ época) y los ciclos cortos sembrados a fines de junio ( $4^{\circ}$ época), mostraron $\boldsymbol{R P R O}$ y $\boldsymbol{R} \boldsymbol{M} \boldsymbol{A} \boldsymbol{X}$ similares.

El análisis presentado omitió la $1^{\circ}$ época de siembra. Para que la fecha de espigazón no resulte posterior al 1 -sep. (fecha para la cual la $\boldsymbol{F U H}$ es $\leq 10 \%$; Fig. 2f), los cultivares de ciclo largo deberían sembrarse en la primer quincena de mayo. Sin embargo, esto resulta poco práctico por varios motivos. Primero, si el cultivo antecesor fuese soja, la siembra de trigo se superpondría con la cosecha de la soja. Segundo, si el cultivo antecesor fuera girasol o maíz, la siembra del trigo podría realizarse en fecha apropiada, pero si esta se retrasara por lluvias, existiría el riesgo de que el cultivo no florezca, ya que muchos de los cultivares de ciclo largo tiene requerimientos de vernalización, que no sería satisfechos en una siembra tardía en Reconquista. Este problema no se presenta en los cultivares de ciclo intermedio y cortos ya que la mayoría de estos cultivares no poseen requerimientos de vernalización relevantes. Tercero, si la siembra de los cultivares de ciclo largo se concretara en la fecha apropiada, quedarían por más tiempo expuestos a estrés biótico y abiótico; en consecuencia, no es de esperar que lleguen con más área verde a las etapas críticas del cultivo que los cultivares de ciclo más corto sembrados más tarde. El tiempo disponible para producir macollos y área verde en un cultivar de ciclo corto es una desventaja, pero ese problema se compensa con el aumento de la densidad al retrasar la fecha de siembra. De hecho, en el norte santafesino la superficie sembrada con cultivares de ciclo largo e intermedio-largo en la última campaña fue baja, $26 \%$ (Bolsa de Cereales, 2019).
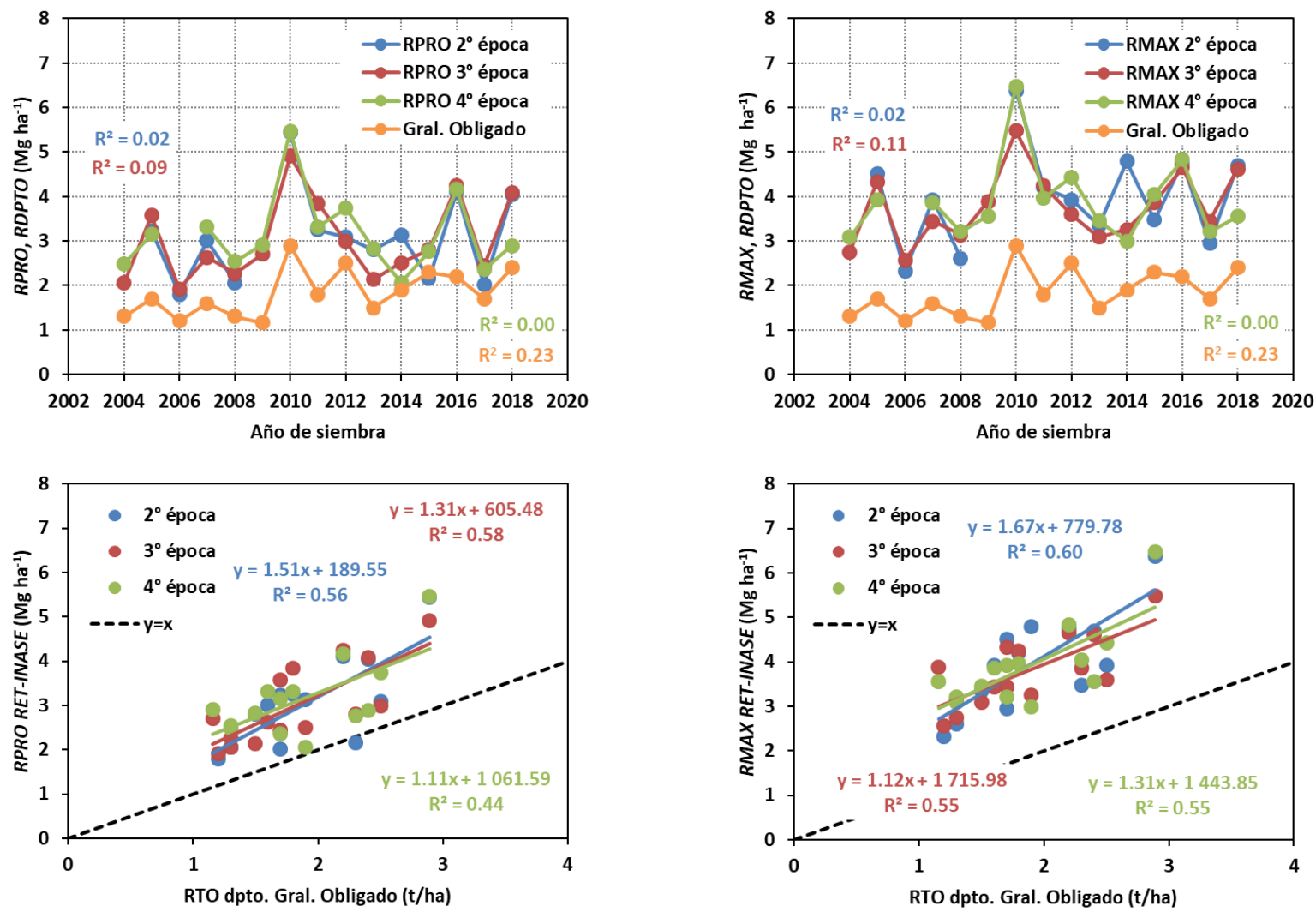

Figura 4. (a) Evolución del rendimiento promedio $(\boldsymbol{R} P \boldsymbol{R} \boldsymbol{O})$ y (b) máximo $(\boldsymbol{R M A X})$ para cada época de siembra de la RET-INASE de INTA Reconquista y rendimiento del departamento de General Obligado (RDPTO); (c) asociación entre el $\boldsymbol{R} \boldsymbol{P R \boldsymbol { O }}$ y (d) $\boldsymbol{R M A X}$, de cada época de siembra de la RET vs. el $\boldsymbol{R} \boldsymbol{T} \boldsymbol{O}$ del departamento de General Obligado, a través de los años. En las figuras (a) y (b) se presenta el coeficiente de determinación $\left(R^{2}\right)$ entre el $\boldsymbol{R P R O}$ o $\boldsymbol{R M} \boldsymbol{A} \boldsymbol{X}$ y el año de siembra, ninguno fue estadísticamente significativo $(P>0.05)$. La mínima diferencia significativa para cada año se presenta en la Tabla 1.

El RPRO y el $\boldsymbol{R M A X}$ de cada épocas de siembra se asoció con el $\boldsymbol{R T O} \boldsymbol{O}$ el dpto. de Gral. Obligado $(P \leq$ 0.01; Fig. 4c y Fig. 4d) denotando que los datos de la RET-INASE de INTA Reconquista resultaron representativos del $\boldsymbol{R}$ TO departamental. De la Fig. 4 surge que, entre los años 2004-2018, el $\boldsymbol{R}$ TO obtenido en el dpto. de 
Gral. Obligado, en condiciones comerciales de producción y con la tecnología aplicada por el productor, promedió $1.83 \mathrm{Mg} \mathrm{ha}^{-1}$ sin tendencia definida a través de los años. Por su parte, el $\boldsymbol{R} T \boldsymbol{O}$ obtenido en la RETINASE de INTA Reconquista con un manejo mejorado, alcanzó un promedio de $3.03 \mathrm{Mg} \mathrm{ha}^{-1}$ y máximo promedio de $4.11 \mathrm{Mg} \mathrm{ha}^{-1}$ (Tabla 1), lo cual implica que la $\boldsymbol{B P R O}$ y la $\boldsymbol{B M A X}$ fueron 66 y $125 \%$ respectivamente.

En la Fig. 5 se presenta la $\boldsymbol{D R P R O}$ y la $\boldsymbol{D R M A X}$ acorde a la Ec.[13]. Si el mejoramiento genético hubiera tenido un impacto continuo y significativo en Reconquista durante los últimos años, debería observarse un aumento de las diferencias de $\boldsymbol{R} \boldsymbol{T} \boldsymbol{O}$ a través de los años, i.e. una línea con pendiente positiva. Sin embargo, todas las pendientes de las rectas de la Fig. 5 estuvieron muy lejos de resultar estadísticamente distintas de cero. Podría presumirse que la falta de tendencias en la Fig. 5 se podría deber a la existencia de una alta interacción entre los cultivares testigos y los cultivares restantes $\mathrm{x}$ año. De hecho, la interacción de todos los cultivares $\mathrm{x}$ año en el análisis por bienios resultó estadísticamente significativa para todos los bienios, excepto 2/36 casos (Tabla 1).

No obstante, se encontró que la diferencia de rendimiento entre los cultivares testigos y los cultivares restantes no se modificó entre años excepto en 4/27 casos (Tabla 1), por lo cual no hay razón para suponer que el resultado de la Fig. 5 sea viciado por una alta interacción entre los cultivares testigos y los cultivares restantes. En coincidencia con la Fig. 5, la Tabla 3 muestra una lista parcial de los cultivares evaluados en más de tres campañas, de la cual no surge correspondencia entre la $\boldsymbol{D R T O}$ y el año de inscripción. Generalizando el análisis para todos los cultivares, la asociación entre la $\boldsymbol{D R T O}$ y el año de inscripción no fue estadísticamente significativa en ninguna de las épocas de siembra: $R^{2}=0.04(\mathrm{gl}=48, P>0.18), R^{2}=0.01(\mathrm{gl}=63, P>0.53), R^{2}=$ $0.07(\mathrm{gl}=42, P>0.08)$, para la $2^{\circ}, 3^{\circ}$ y $4^{\circ}$ época, respectivamente.
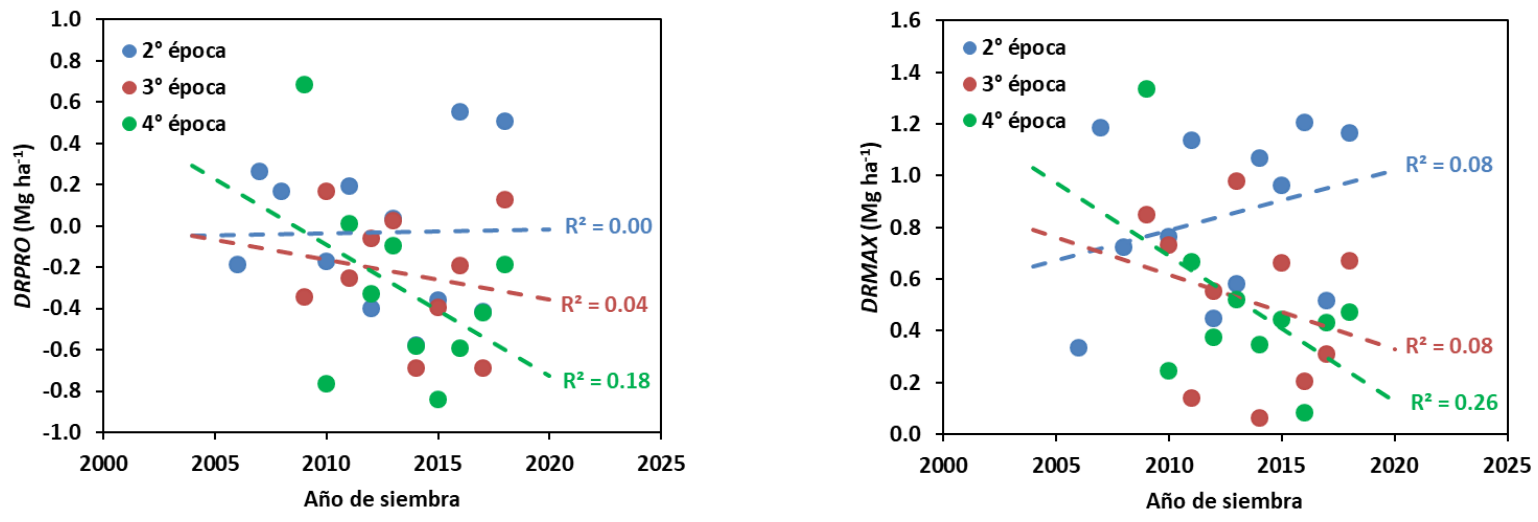

Figura 5. Diferencia entre el (a) rendimiento promedio (DRPRO, Ec.[13]) y el (b) rendimiento máximo $(\boldsymbol{D R} \boldsymbol{M} \boldsymbol{A} \boldsymbol{X})$, respecto del rendimiento de los cultivares testigos (Tabla 2), para cada época de siembra de la RET-INASE de INTA Reconquista a través de los años. La mínima diferencia significativa para comparar cultivares en cada año se presenta en la Tabla 1. Las líneas punteadas no fueron significativas $(P>0.05)$.

Una primera pregunta que sigue a la conclusión proveniente de la Fig. 5 y la Tabla 3 es si el mejoramiento genético reciente fue exitoso en alguna zona de Argentina. Lo Valvo et al. (2018) al analizar el progreso genético de trigo en Argentina desde 1918 al 2011, encontraron que entre 1999 y 2011 la tasa de progreso fue $14 \mathrm{~kg}$ $\mathrm{ha}^{-1} \operatorname{años}^{-1}\left(0.18 \%\right.$ año $\left.{ }^{-1}\right)$, tasa compatible con el escaso progreso observado en la Fig. 5 y la Tabla 3. Por otra parte, puede mencionarse que haciendo un análisis similar al de la Fig. $5 \mathrm{~b}$ con la $1^{\circ}$ época de siembra con fungicida de la RET-INASE de INTA Balcarce entre los años 2006 y 2018, se encontró una clara asociación positiva $\left(R^{2}=0.61, P \leq 0.001\right)$. Este avance en el mejoramiento sería atribuible a la incorporación de "sangre francesa" a los cultivares argentinos de ciclo largo a partir de la introducción del cultivar Baguette 10 en el año 2000 (Abbate et al., 2017). Sin embrago, en el norte de Santa Fe, los cultivares de ciclo largo imponen restricciones en la secuencia de cultivos, por lo cual un avance genético en cultivares de ese ciclo no tendría un impacto tan grande como más al sur. Por otra parte, los cultivares con germoplasma francés suelen ser más susceptibles a las enfermedades foliares que los cultivares tradicionales. La mayor dependencia de estos cultivares a la aplicación de fungicida limitaría su difusión en el norte de Santa Fe ya que la expectativa de rendimiento de trigo es menor que más al sur, por lo cual el uso de fungicidas es poco generalizado. De hecho los cultivares de ciclo más largo, participantes de la $1^{\circ}$ y $2^{\circ}$ época de siembra de la RET-INASE de INTA Reconquista, no 
Abbate, P.E. y Brach, A.M.: Trigo: cambio climático y mejoramiento genético.

mostraron ventajas de $\boldsymbol{R} \boldsymbol{T O}$ respecto de los cultivares de ciclo corto participantes de la $3^{\circ}$ y $4^{\circ}$ época (Tabla 2 , Fig. 4). Por lo cual no hay evidencias de que el mejoramiento genético basado en la incorporación de germoplasma francés haya resultado exitoso en el norte de Santa Fe.

Tabla 3. Listado de los 20 cultivares que presentaron mayor diferencias de rendimiento promedio (DRTO, Ec. [14]) respecto de los cultivares testigos (Tabla 2), evaluados tres o más años, en cada época de siembra y sus principales características: año de inscripción, primer y último año de participación en la RET-INASE de INTA Reconquista. La mínima diferencia significativa para comparar cultivares dentro de cada experimento se presenta en la Tabla 1.

\begin{tabular}{|c|c|c|c|c|c|c|c|c|c|c|c|c|c|c|}
\hline \multicolumn{5}{|c|}{$2^{\circ}$ época } & \multicolumn{5}{|c|}{$3^{\circ}$ época } & \multicolumn{5}{|c|}{$4^{\circ}$ época } \\
\hline Cultivar $^{\mathrm{a}}$ & Inscrip. & Primer & Ultimo & $\begin{array}{l}\text { DRTO } \\
\left(\mathrm{Mg} \mathrm{ha}^{-1}\right)\end{array}$ & Cultivar $^{\mathrm{a}}$ & Inscrip. & Primer & Ultimo & $\begin{array}{l}\text { DRTO } \\
\left(\mathrm{Mg} \mathrm{ha}^{-1}\right)\end{array}$ & Cultivar ${ }^{\mathrm{a}}$ & Inscrip. & Primer & Ultimo & $\begin{array}{l}\text { DRTO } \\
\left(\mathrm{Mg} \mathrm{ha}^{-1}\right)\end{array}$ \\
\hline ACA 602 & 2013 & 2013 & $>2018^{b}$ & 0.62 & K. CASTOR & 2005 & 2005 & 2009 & 0.50 & BAG. P. 13 & 2001 & 2004 & 2009 & 0.59 \\
\hline MS INTA 415 & 2016 & 2016 & $>2018$ & 0.61 & ACA 602 & 2013 & 2013 & $>2018$ & 0.30 & LE 2331 & 2009 & 2008 & 2015 & 0.38 \\
\hline K. LIEBRE & 2013 & 2015 & $>2018$ & 0.55 & ONIX & 2004 & 2004 & 2009 & 0.29 & K. CASTOR & 2005 & 2005 & 2009 & 0.18 \\
\hline SY 200 & 2010 & 2010 & $>2018$ & 0.42 & B. SAETA & 2015 & 2015 & $>2018$ & 0.29 & AGP FAST & 2009 & 2009 & 2014 & 0.17 \\
\hline K. PROMETEO & 2016 & 2016 & $>2018$ & 0.35 & BAG. P. 13 & 2001 & 2004 & 2009 & 0.28 & BIO. 1005 & 2008 & 2008 & 2015 & 0.12 \\
\hline K. GUERRERO & 2007 & 2007 & 2013 & 0.29 & K. LANZA & 2016 & 2016 & $>2018$ & 0.27 & K. LANZA & 2016 & 2016 & $>2018$ & 0.06 \\
\hline K. GAVILÁN & 2004 & 2004 & 2007 & 0.28 & B. PLENO & 2012 & 2012 & $>2018$ & 0.26 & K. TAURO & 2005 & 2005 & $>2018$ & 0.04 \\
\hline B. ARRIERO & 1998 & 2005 & 2008 & 0.21 & K. TAURO & 2005 & 2005 & $>2018$ & 0.24 & ACA 901 & 2006 & 2006 & 2012 & 0.04 \\
\hline BIO. 2002 & 2006 & 2006 & 2008 & 0.20 & CRONOX & 2005 & 2005 & 2013 & 0.24 & K. TIGRE & 2008 & 2008 & 2011 & 0.03 \\
\hline ACA 601 & 2003 & 2004 & 2009 & 0.15 & LE 2331 & 2009 & 2008 & 2015 & 0.22 & K. NUTRIA & 2009 & 2009 & $>2018$ & 0.03 \\
\hline $\begin{array}{l}\text { K. TITANIO } \\
\text { CL }\end{array}$ & 2015 & 2015 & $>2018$ & 0.15 & ACA 801 & 2004 & 2004 & 2008 & 0.18 & K. ZORRO & 2006 & 2006 & 2012 & 0.00 \\
\hline $\begin{array}{l}\text { B. CHACARE- } \\
\text { RO }\end{array}$ & 2005 & 2005 & 2008 & 0.12 & AREX & 2010 & 2010 & 2013 & 0.18 & SY 330 & 2015 & 2015 & $>2018$ & -0.02 \\
\hline B. MATACO & 2002 & 2004 & 2008 & 0.11 & LE 2294 & 2005 & 2005 & 2008 & 0.17 & ONIX & 2004 & 2004 & 2009 & -0.02 \\
\hline BIO. 1002 & 2005 & 2005 & 2011 & 0.11 & K. FLECHA & 2003 & 2004 & 2006 & 0.17 & ACA 801 & 2004 & 2004 & 2008 & -0.07 \\
\hline K. PROTEO & 2003 & 2004 & $>2018$ & 0.09 & ATLAX & 2008 & 2009 & 2011 & 0.12 & BIO. 1006 & 2009 & 2009 & $>2018$ & -0.08 \\
\hline BIO. 3000 & 2004 & 2005 & 2011 & 0.06 & B. CLARAZ & 2016 & 2016 & $>2018$ & 0.12 & ACA 903 B & 2008 & 2008 & 2012 & -0.10 \\
\hline ACA 360 & 2013 & 2013 & $>2018$ & 0.05 & AGP FAST & 2009 & 2009 & 2014 & 0.12 & K. LEÓN & 2009 & 2009 & 2016 & -0.11 \\
\hline ACA 302 & 2002 & 2004 & 2007 & 0.04 & BIO. 1005 & 2008 & 2008 & 2015 & 0.10 & K. CHAJÁ & 2002 & 2004 & 2011 & -0.13 \\
\hline K. JABALÍ & 2002 & 2005 & 2007 & 0.03 & K. LEÓN & 2009 & 2009 & 2016 & 0.09 & BIO. 1001 & 2004 & 2005 & 2009 & -0.13 \\
\hline ALGARROBO & 2015 & 2015 & $>2018$ & 0.01 & B. AGUARÁ & 2004 & 2004 & 2006 & 0.06 & CRONOX & 2005 & 2005 & 2013 & -0.16 \\
\hline
\end{tabular}

${ }^{a}$ B., Buck; BAG., Baguette; BIO., BIOINTA/BIOCERES; BON., Bonaerense; K., Klein; P., Premium.

b $>2018$, implica que continuaba siendo comercializado en ese año.

Si bien no se encontró evidencia de que hubiera un avance continuo del mejoramiento genético en Reconquista (Fig. 5), pudo haber un progreso ocasional mediante la introducción en la zona cultivares de RTO destacado. La Tabla 3 lista, para cada época se siembra, los 20 cultivares que presentaron la mayor DRTO (Ec.[14]). Los cinco primeros cultivares listados para $2^{\circ}$ época de siembra son comercializados actualmente. En la $3^{\circ}$ época solamente dos cultivares actualmente comerciales están entre los cinco de mayor DRTO. La $4^{\circ}$ época presenta la situación más desfavorable, el primer cultivar aun comercial ocupa el sexto lugar y ninguno de los cultivares comercializados actualmente supera el RTO de los cultivares testigos. En vista de la Tabla 3 resulta entendible que entre los cultivares con más difusión en el norte de Santa Fe se incluyan ACA 602 (inscripto en el 2013), Klein Tauro (2005), Klein Nutria (2009) y SY 200 (2010) ya que son los cultivares actualmente comercializados con mayor DRTO.

\section{CONCLUSIONES}

El presente trabajo comenzó observando que a partir del año 2000 la tasa promedio de aumento de $\boldsymbol{R T O}$ de trigo pan en Argentina se elevó a $46.0 \mathrm{~kg} \mathrm{ha}^{-1}$ año ${ }^{-1}$, mientras que el norte de Santa Fe mantuvo una tasa de 19.4 $\mathrm{kg} \mathrm{ha}^{-1} \mathrm{año}^{-1}$ desde el comienzo de los años 1970. Para indagar sobre el origen de esa diferencia, se analizó si en las dos últimas décadas hubo algún cambio climático relevante, cuál fue la brecha tecnológica y cuál fue el aporte del mejoramiento genético al cultivo, en el norte de Santa Fe. 
Los datos de la estación meteorológica de INTA Reconquista mostraron que hubo cambios en las principales variables climáticas entre los años recientes (2000-2018) y los previos (1971-1999), durante el período de cultivo de trigo (1-may. al 31-dic.). El cambio más desfavorable fue el aumento del $\boldsymbol{D} \boldsymbol{P} \boldsymbol{V}_{\boldsymbol{P}}$ promediado desde 20 d antes a 10 d después del 1-sep. (fecha óptima de espigazón). En definitiva, pudo estimarse que los cambios climáticos implicarían una reducción de hasta el $51 \%$ en la tasa de aumento de $\boldsymbol{R T O}$ en el norte de Santa Fe desde el año 2000 a 2018.

El RPRO y el $\boldsymbol{R M A X}$ anual de todas las épocas de siembra de la RET-INASE de INTA Reconquista se asoció con el RTO el departamento de Gral. Obligado indicando que los datos experimentales resultan representativos del $\boldsymbol{R} \boldsymbol{T} \boldsymbol{O}$ departamental. No obstante, la $\boldsymbol{B P R O}$ y la $\boldsymbol{B M} \boldsymbol{A} \boldsymbol{X}$ entre el $\boldsymbol{R} \boldsymbol{T} \boldsymbol{O}$ departamental y el obtenido en la RET-INASE de INTA Reconquista fueron de 66 y $125 \%$ respectivamente.

La oferta de cultivares en el norte de Santa Fe es amplia, de hecho en la RET-INASE de INTA Reconquista se evaluó un promedio de 45 cultivares por años desde 2004. Sin embargo, no se encontró evidencia de avance genético continuo en los últimos 10-13 años. No obstante, se identificaron cultivares comercializados actualmente de $\boldsymbol{R} \boldsymbol{T} \boldsymbol{O}$ destacado, independientemente de su año de introducción. El escaso avance genético logrado en la zona podría deberse a que la incorporación de germoplasma francés, predominantemente en cultivares de ciclo largo, no haya resultado exitosa en el norte de Santa Fe donde los cultivares de ciclo corto se adaptan mejor a la rotación con soja (cultivo predominante). Un mayor avance genético podría impulsar la difusión del trigo para superar el 22\% de superficie agrícola del norte de Santa Fe que ocupa actualmente.

\section{BIBLIOGRAFÍA}

Abbate, P.E. (2017). Capítulo 3: Bases ecofisiológicas del manejo del cultivo de trigo en la región pampeana. En: Divito A. y García F. O. (Eds.), Manual del cultivo de trigo. Instituto Internacional de Nutrición de Plantas Programa Latinoamérica Cono Sur (IPNI), Acassuso, Buenos Aires, Argentina. Págs. 33-52.

Abbate, P.E., Aguate, F.M., Del Vecchio, E., y Balzarini, M. (2017). Estabilidad del rendimiento interanual en INTA Balcarce, campañas 2013/14 a 2016/17. INTA, EEA Balcarce. https://inta.gob.ar/ sites/default/files/inta ret balcarce 2016 estabilidad.pdf. Último acceso, 7-ene-2020.

Abbate, P.E., Dardanelli, J.L., Cantarero, M.G., Maturano, M., Melchiori R.J.M., y Suero, E.E. (2004). Climatic and water availability effects on water use efficiency in wheat. Crop Science 44 (2): 474-483. https://doi.org/10.2135/cropsci2004.4740

Abbate, P.E. y Lázaro L. (2010). Estimación del rendimiento potencial de trigo en Paraguay y sus requerimientos de agua y nutrientes. En: Kholi, M.M., Cubilla L.E. y Cabrera G. (Eds.), Tercer Seminario Nacional de Trigo: Del Grano al Pan. 2010. CAPECO/INBIO, Asunción, Paraguay. Págs. 11-29. https://capeco.org.py/wp-content/uploads/2015/06/3er-seminario-nacional-del-trigo.pdf

Allen, R.G., Pereira, L.S., Raes, D. y Smith, M. (1998). Crop evapotranspiration: Guidelines for computing crop water requirements. Irrigation and Drainage Paper 56. FAO, Rome, Italy, 300 pp.

Bolsa de Cereales. (2019). Relevamiento de tecnología agrícola aplicada: campaña 2018/19. Bolsa de Cereales, Buenos Aires. $\quad \underline{\mathrm{http}}$ ://www.bolsadecereales.com/descargar-documento2-196/retaa84372a6c91d53035d612d39c124bb7fb. Último acceso, 7-ene-2020.

Brach, A.M. (2011). Validación del cociente fototermal como predictor del rendimiento potencial de diferentes genotipos de trigo en el norte de la provincia de Santa Fe. Tesis Magister, Facultad de Ciencias Agrarias, Universidad Nacional del Nordeste, Corrientes, Argentina.

Cantarero, M.G., Abbate, P.E., Balzarini, S.M. (2016). Effect of water stress during the spike growth period on wheat yield in contrasting weather. International Journal of Environmental \& Agriculture Research 2 (7), 22-37.

Caviglia O.P., Abbate P.E., Paparotti O.F., Melchiori R.J. y Barbagelata P.A. (2001). Determinación del rendimiento del trigo en Paraná: una comparación con Balcarce. Actas del V Congreso Nacional de Trigo. Villa Carlos Paz, Córdoba.

DEAD (Dirección de Estimaciones Agrícolas y Delegaciones del Ministerio de Agricultura, Ganadería y Pesca, Argentina). (2019). http://datosestimaciones.magyp.gob.ar/. Último acceso, 7-ene-2020. 
Abbate, P.E. y Brach, A.M.: Trigo: cambio climático y mejoramiento genético.

Fischer, R.A, (1985). Number of kernels in wheat crops and the influence of solar radiation and temperature. The Journal of Agricultural Science, 105 (2), 447-461. https://doi.org/10.1017/ S0021859600056495

Fischer, R.A., Byerlee D. y Edmeades, G.O. (2014). Wheat. Crop yields and global food security: will yield increase continue to feed the world? ACIAR Monograph $\mathrm{N}^{\circ} 158$. Australian Centre for International Agricultural Research, Canberra: 65-132.

Ghanem, M.E., Kehel, Z., Marrou, H., Sinclair, T.R. (2020). Seasonal and climatic var iation of weighted VPD for transpiration estimation. European Journal of Agronomy, 113: 125966. https:// doi.org/10.1016/j.eja.2019.125966

Giorgi, R., Tosolini, R., Sapino, V., León, C. y Chiavassa, A. (2009). Evaluación de la capacidad productiva de las tierras de la provincia de Santa Fe para uso agrícola y pasturas de alfalfa. http:// rafaela.inta.gob.ar/mapas/capacidad_productiva/index.htm. Último acceso, 7-ene-2020.

Kemanian, A.R., Stökle, C.O., y Huggins, D.R. (2004). Variability of barley radiation use efficiency. Crop Science, 44: 1662-1672. https://doi.org/10.2135/cropsci2004.1662

Lázaro, L. y Abbate, P.E. (2012). Cultivar effects on relationship between grain number and photothermal quotient or spike dry weight in wheat. Journal of Agricultural Science, 150 (4): 442-459. https:// doi.org/10.1017/S0021859611000736

Lo Valvo, P.J., Miralles, D.J., Serrago, R.A. (2018). Genetic progress in Argentine bread wheat varieties released between 1918 and 2011: Changes in physiological and numerical yield components. Field Crops Research, 221: 314-321. https://doi.org/10.1016/j.fcr.2017.08.014

McIntosh, M.S. (1983). Analysis of combined experiments. Agron. J., 75:153-155.

USDA (Departamento de Agricultura de los Estados Unidos) Soil Survey Staff. (2014). Claves para la Taxonomía de Suelos. $12^{\circ}$ Edición. USDA, Natural Resources Conservation Service, 142. Washington, DC, $410 \mathrm{pp}$.

SAGPyA (Secretaría de Agricultura, Ganadería, Pesca y Alimentación). (2004). Resolución 1262. Norma de Calidad para la Comercialización de Trigo Pan - Norma XX Trigo Pan. Boletín Oficial de la República Argentina, Primera Sección, Secretaría Legal y Técnica, Dirección Nacional del Registro Oficial, 30550,6 .

Steel, R.G.D. y Torrie, J.H. (1980). Bioestadística, principios y procedimientos. 1st ed. en español. McGraw-Hill, México.

Tanner, C.B. y Sinclair, T.R. (1983). Efficient water use in crop production: Research or re-research? En: Taylor et al. (Eds.) Limitations to efficient water use in crop production. ASSA, CSSA, SSSA, Madison, WI. Págs. 1-27. https://doi.org/10.2134/1983.limitationstoefficientwateruse.c1

Vidal, C.M. (2006). Evaluación de la aptitud de las tierras para riego por aspersión en el Noreste Santafesino. Tesis Especialización en riego de tierras agrícolas. Universidad Nacional del Litoral. 